\title{
An equivalent modeling method for Offshore Wind Farms based on fault characteristics analysis
}

\author{
Haiyan Tang ${ }^{1, *}$, Guanglei $\mathrm{Li}^{2}$, Linan $\mathrm{Qu}^{3}$, and Yan $\mathrm{Li}^{1}$ \\ ${ }^{1}$ State Key Laboratory of Operation and Control of Renewable Energy \& Storage Systems, China Electric Power Research Institute, \\ Beijing, China \\ ${ }^{2}$ State Grid Shandong Electric Power Company, Jinan, China \\ ${ }^{3}$ State Key Laboratory of Operation and Control of Renewable Energy \& Storage Systems, China Electric Power Research Institute, \\ Nanjing, China
}

\begin{abstract}
A large offshore wind farm usually consists of dozens or even hundreds of wind turbines. Due to the limitation of the simulation scale, it is necessary to develop an equivalent model of offshore wind farms for power system studies. At present, the aggregation method is widely adopted for wind farm equivalent modeling. In this paper, the topology, electrical parameters, operating conditions and individual turbine characteristics of the offshore wind farms are taken into consideration. Firstly, the output power distribution of offshore wind farm, the voltage distribution of the collector system and the fault ride-through characteristics of wind turbines are analyzed. Then, a dynamic equivalent modeling method for offshore wind farms is developed based on the fault characteristics analysis. Finally, the proposed method is validated through time-domain simulation.
\end{abstract}

\section{Introduction}

Offshore wind farms (OWFs) have broad prospects and space for development [1]. With the development and maturity of offshore wind power technology, the scale of OWFs will become larger. A large OWF usually contains a great number of wind turbines (WTs). In power system time-domain simulation analysis, the utilization of detailed model of each WT will result in massive computation, long time, large memory usage and difficulties in data processing and results analysis [2]. Therefore, it is necessary to establish an equivalent model for stability analysis of OWFs integrated power systems, which could accurately reflect the dynamic characteristics of OWFs under grid faults.

At present, the aggregation method is widely adopted for wind farm equivalent modeling. The wind farm is aggregated into a single or multi-machine equivalent model by preserving the model structure of WT and reducing the number of WTs in the wind farms [3]. The equivalent model and the detailed model of the wind farm have the same voltage, current and power characteristics at the point of common coupling (PCC). To establish an accurate equivalent model of a wind farm, two main steps need to be taken: the cluster division and parameter aggregation of WTs. The cluster division mainly considers the type and operating point of WTs and the studies in which the equivalent model to be applied [4-6]. The equivalent parameter of the generator can be obtained by weighting method or parameter identification [7-8].
The fault ride-through (FRT) characteristics of WTs are closely related to the operating points. The offshore and onshore wind farms have differences in terms of collector system design and wind conditions. In this paper, the output power distribution of OWFs, the voltage distribution of the collector system and the FRT characteristics of WTs are analyzed. Then, a dynamic equivalent modeling method for OWFs is developed based on the fault characteristics analysis. Finally, the effectiveness of the proposed method is verified through time-domain simulation.

\section{Operation characteristics of OWFs}

\subsection{Voltage distribution of collector system}

At present, OWFs usually adopt a two-stage boosting mode. The output voltage of the WT is $690 \mathrm{~V}$ which is boosted to $35 \mathrm{kV}$ by the box-type transformer. Then, the output power of WTs are collected to the $110 \mathrm{kV}$ or $220 \mathrm{kV}$ substation through the $35 \mathrm{kV}$ submarine cable. Finally, the OWF is integrated to the utility grid through the $110 \mathrm{kV}$ or $220 \mathrm{kV}$ line. Generally, the offshore substation has a distance of $20-80 \mathrm{~km}$ with the onshore substation, and the submarine cable is used for transmission. The OWF is arranged in a trunk line. The WTs are divided into groups with each group of WTs connecting to the same feeder cable and $0.5-1 \mathrm{~km}$ distance. The OWF integrated system model is established based on this exemplary topology. The transmission distance of each $220 \mathrm{kV}$ submarine cable is $50 \mathrm{~km}$. The distance of

\footnotetext{
* Corresponding author: tanghy@epri.sgcc.com.cn
} 
each $35 \mathrm{kV}$ cable is $10 \mathrm{~km}$ with $10 \mathrm{WTs}$ integrated. The power factor of WTs in OWF are set to be 1.0. The grid side voltage is running at $1.0 \mathrm{pu}$. The voltage distribution characteristics of the OWF integrated system is shown in Fig. 1. In the wind farm output range, the terminal voltage from the $\# 10$ to $\# 1$ is increasing with $0.010 \mathrm{pu}$ voltage difference.

\subsection{Output power distribution of OWFs}

Compared with onshore wind farms, OWFs are generally unaffected by topographical features. The sea surface is flat and the wind speed is stable, which has a constant dominant wind direction, for most cases. Fig. 2 shows the wind speed variation of WTs at different locations in an OWF. The wind speed is mostly in the range of $7 \mathrm{~m} / \mathrm{s} \sim 10 \mathrm{~m} / \mathrm{s}$.

When the wind speed is low, the WT operates in the maximum power point tracking (MPPT) state. When the wind speed is higher than the rated, the WT operates in the limited power mode with the action of pitch angle control. Figure 3 shows the power curve of a $3 \mathrm{MW}$ WT at standard air density $1.225 \mathrm{~kg} / \mathrm{m}^{3}$. In the range of
$7 \mathrm{~m} / \mathrm{s} \sim 10 \mathrm{~m} / \mathrm{s}$, the WT output power is about $0.7 \mathrm{pu} \sim 1 \mathrm{pu}$. Therefore, WTs in the OWF have a smaller ratio of the output fluctuation to installed capacity than the onshore wind farm. The output power of the WTs at different locations of an OWF is relatively closer than the onshore wind farm.

\subsection{FRT characteristics of WT}

Different types of WTs adopt different low-voltage ridethrough (LVRT) control strategies and parameters, which will show different FRT characteristics under grid faults. However, according to the process of grid fault occurrence and clearance, the basic form of active and reactive characteristics of WTs under grid faults is as shown in Fig. 4. Three steps are included: the period of LVRT, recovery start and recovery process. During the period of LVRT, the WT outputs a certain dynamic reactive power to support the grid voltage. After the clearance of the fault, the WT reactive power gradually stabilizes at the initial value after a transient process. In addition, the FRT characteristics of the WT IS related to the initial output state.

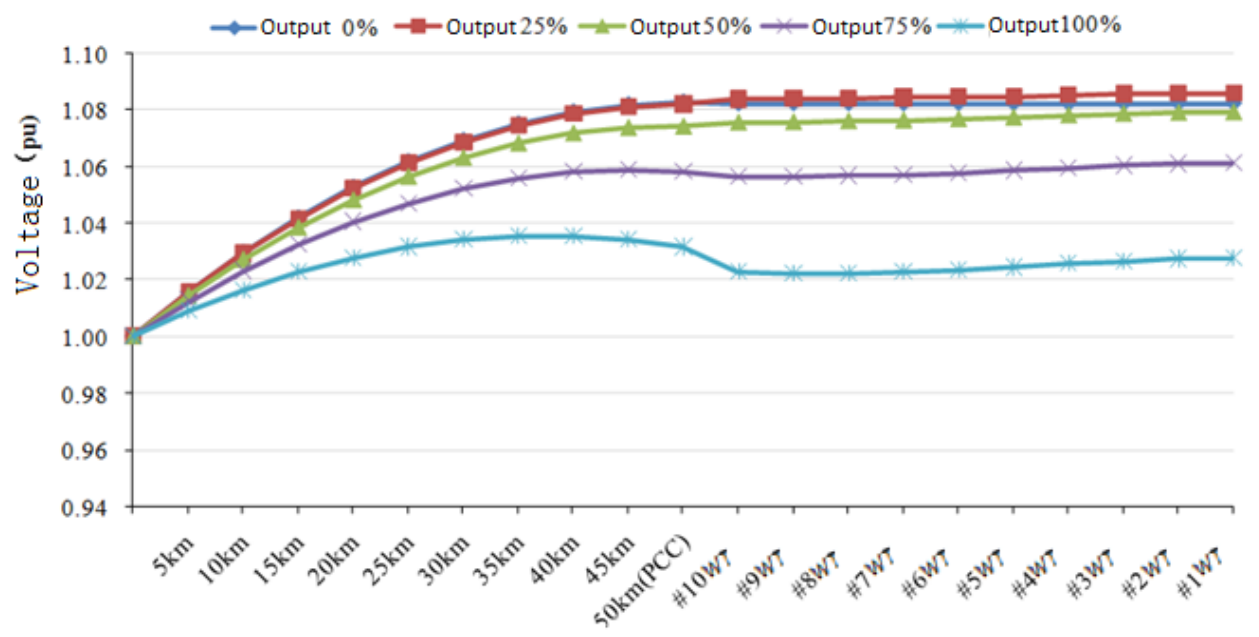

Fig. 1. Voltage distribution of OWF integrated system.

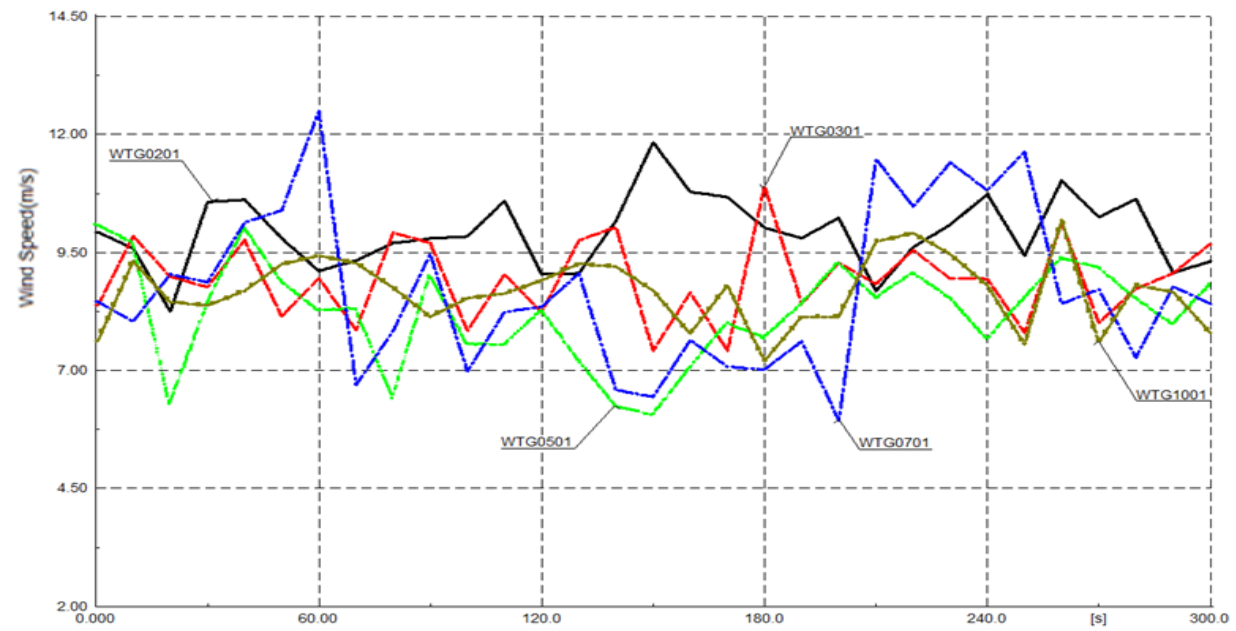

Fig. 2. Wind speed variation of WTs in an OWF. 


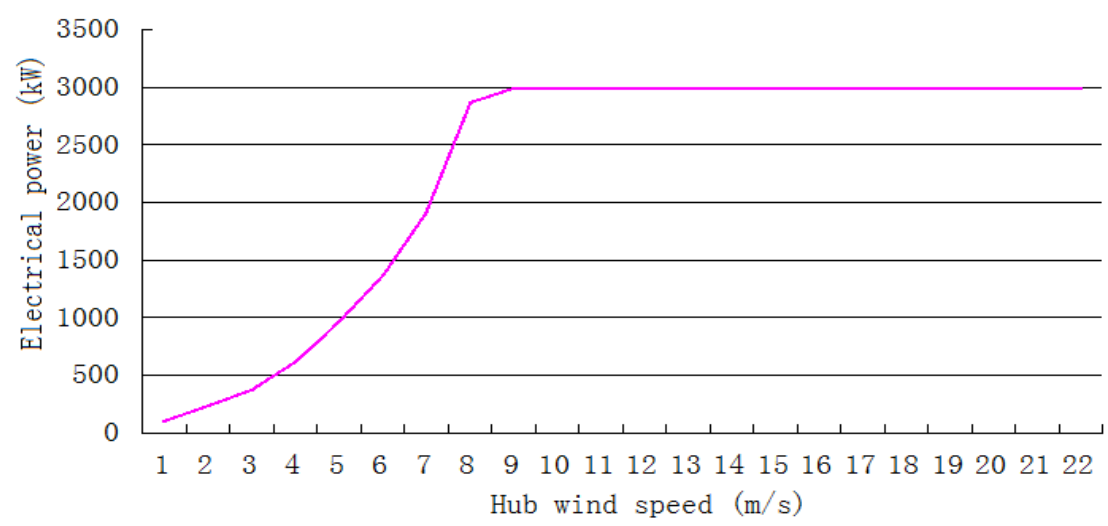

Fig. 3. Power curve of a WT at standard air density.
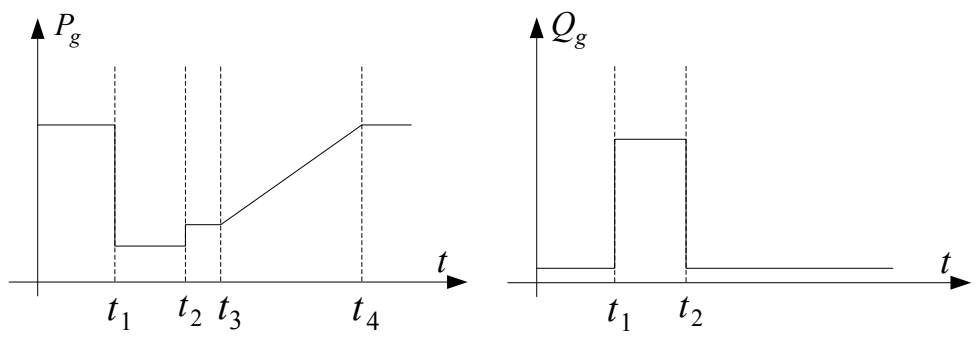

Fig. 4. Basic form of active and reactive power characteristics of WTs under grid fault.

\section{Equivalent modeling method based on fault characteristics analysis}

\subsection{Equivalent method}

When the aggregation method is used to equivalent modeling of OWFs, the division of the cluster is firstly carried out. The main considerations include the output power distribution of the OWF, the voltage distribution of the collector system and the FRT characteristics of the WT.

(1) The voltage distribution of WTs on the same feeder of an OWF is relatively close. Therefore, in principle, WTs of one feeder can be grouped to one cluster. For special cases where the feeder is long with many wind turbines connected, WTs of one feeder can be grouped to multiple clusters based on the voltage distribution.

(2) According to the FRT characteristics analysis, the required number of equivalent machines for the divided clusters is determined. The WT under grid fault is simulated to analyze the FRT characteristics. The FRT process is divided into three stages: pre-fault (A), fault period (B) and post-fault (C) according to the fault time sequence. The active and reactive power average absolute deviation of three stages are calculated as follows:

$$
F=\frac{\sum_{i=\mathrm{K}_{\text {Sert }}}^{\mathrm{K}_{\mathrm{End}}}\left|\left(X_{M}(i)-X_{S}(i)\right)\right|}{\mathrm{K}_{\mathrm{End}}-\mathrm{K}_{\text {Start }}+1}
$$

where, $\mathrm{X}_{\mathrm{M}}$ is the power simulation value of each WT in the cluster at the actual wind speed, $X_{S}$ is the weighted average value of the initial operating power of all WTs. $\mathrm{K}_{\text {Start }}$ and $\mathrm{K}_{\text {End }}$ respectively indicate the sequence numbers of the data for each period of A, B, and C.

The weighted average absolute deviation of the active and reactive power is calculated as follows:

$$
\begin{aligned}
& F_{G_{-} P}=K_{A} * F_{A P}+K_{B} * F_{B P}+K_{C} * F_{C P} \\
& F_{G_{-} Q}=K_{A} * F_{A Q}+K_{B} * F_{B Q}+K_{C} * F_{C Q}
\end{aligned}
$$

where $K_{A}, K_{B}$ and $K_{C}$ are weighting coefficients, generally can be taken as $0.1,0.6$ and 0.3 . The size of $F_{G_{-} P}$ and $F_{G_{-} Q}$ determines the number of equalizers in each cluster in step (1). The smaller the value $F_{G_{-} P}$ and $F_{G_{-}}$, the higher the equivalent precision.

(3) Performing wind speed configuration to determine the initial operating power of the equivalent machine as the weighted average of the initial operating power of all wind turbines.

(4) After determining the principle of the cluster division of OWFs, the parameters are aggregated for the WT and the wind farm collector system. Finally, the equivalent model is compared with the detailed model.

The flow chart of the equivalent modeling of OWFs based on fault characteristics analysis is shown in Fig. 5. 


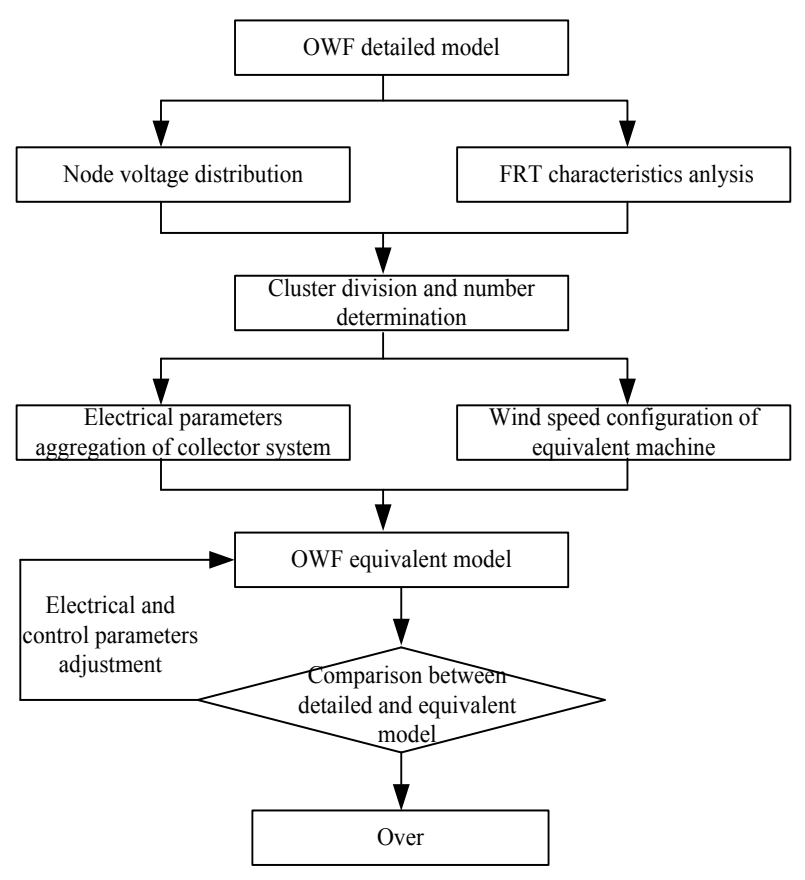

Fig. 5. Flow chart of equivalent modeling of OWFs based on fault characteristics analysis.

\subsection{Simulation analysis}

The structure of the OWF detailed model used in the case study is shown in Fig. 6. The WTs are all full-power wind turbines. According to the voltage distribution analysis, feeder 1 and feeder 2 are each divided into a cluster. Based on wind speed distribution and FRT characteristics analysis, cluster feeder 1 is aggregated into one equivalent machine and cluster feeder 2 is aggregated into two equivalent machines. Therefore, the OWF equivalent model consists of three machines. The comparison results between the detailed model and the equivalent model are shown in Fig. 7. The simulation results show that the equivalent model can accurately reflect the dynamic characteristics of the detailed model of the OWF under grid fault.

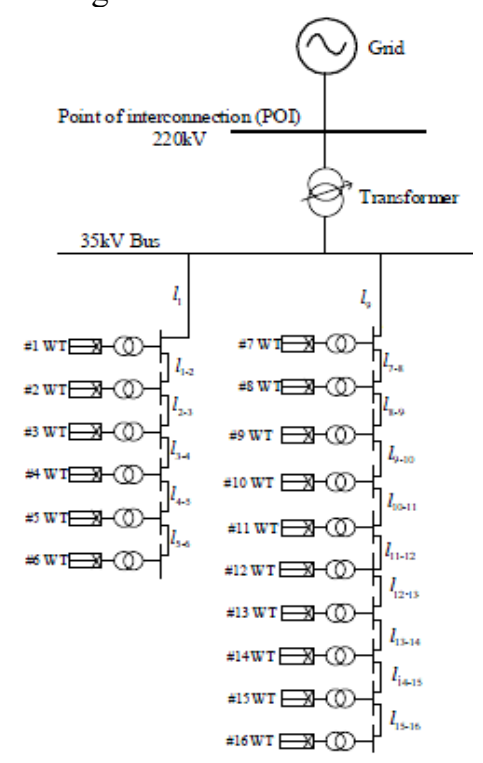

Fig. 6. Structure of OWF detailed model.

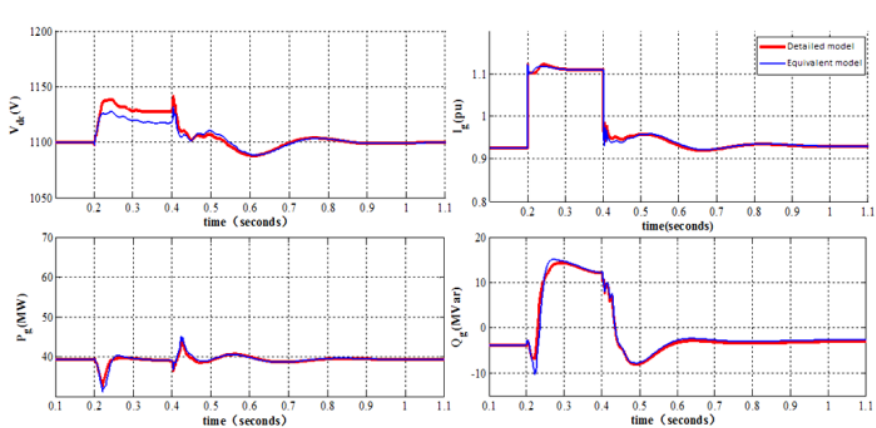

(a) DC voltage (b) converter output current (c) OWF active power (d) OWF reactive power

Fig. 7. Comparison of detailed and equivalent model of OWF under grid fault.

\section{Conclusions}

In this paper, the cluster division in the equivalent modeling of OWFs is studied. Some analytical conclusions are given, including that the output power of the WTs at different locations of an OWF is relatively close, the voltage distribution of WTs on the same feeder of an OWF is relatively close, and different types of WTs show different FRT characteristics under grid faults. Taking into account the output power distribution of the OWF, the voltage distribution of the collector system and the FRT characteristics of the WT, a dynamic equivalent modeling method for OWFs based on fault characteristics analysis is proposed. Finally, the effectiveness of method is validated through the time-domain simulation.

This work was supported by Science and technology project of State Grid Corporation of China (Research and development of control and protection technology for flexible DC transmission of large-scale offshore wind power base).

\section{References}

1. Chi Yongning, Liang Wei, Zhang Zhankui, et al.An Overview on Key Technologies Regarding Power Transmission and Grid Integration of Large Scale Offshore Wind Power [J]. Proceedings of the CSEE, 2016, 36(14):3758-3771.

2. Zhang Yuan, Hao Lili, Dai Jiaqi. Overview of the equivalent model research for wind farms [J].Power System Protection and Control, 2015, 43(06):138146.

3. A. Pernama, S. Uski-Joutsenvuo, O. Carlson, et al. Comparison of an aggregated model of a wind farm consisting of fixed-speed wind turbines with field measurement[J].Wind Energy,2008,11(1):13-27.

4. L.M. Fernandez. J.R. Saena, F.Jurado. Dynamic models of wind farms with fixed speed wind turbines [J]. Renewalbe Energy, 2006, 31(8):1203-1230.

5. Zhou Ming, Ge Jiangbei, Li Gengyin. Cloud Model based DFIG Wind Farm Dynamic Voltage Equivalence Method [J].Proceedings of the CSEE, 2015, 35(05):1097-1105. 
6. ALI, M., ILIE, I.-S., MILANOVIC, J. V., et al. Wind Farm Model Aggregation Using Probabilistic Clustering [J]. IEEE Transactions on Power Systems, 2013, 1 (1):309-316.

7. Jin Yuqing, Ju Ping, Pan Xueping. Analysis on Controller Aggregation Method for Equivalent Modeling of DFIG-based Wind Farm [J].Automation of Electric Power Systems, 2014, 38(3):19-24.
8. Mou Pengtao, Zhao Dongmei, Wang Jiacheng. Study on Wind Farm Equivalence Model Based on MMBN Clustering Algorithm and Capacity Weighting Parameter Aggregation Method[J].Journal of Wuhan University of Technology,2015, 37(11):101-106. 